\title{
Axion cooling of white dwarfs
}

\author{
J. Isern ${ }^{1,2}$, S. Catalán ${ }^{3}$, E. García-Berro ${ }^{4,2}$, M. Salaris ${ }^{5}$, S. Torres ${ }^{4,2}$ \\ ${ }^{1}$ Institut de Ciències de l'Espai (CSIC), Campus UAB, 08193 Bellaterra, Spain \\ ${ }^{2}$ Institut d'Estudis Espacials de Catalunya (IEEC), Ed. Nexus, c/Gran Capità, 08034 Barcelona, \\ Spain \\ ${ }^{3}$ Center for Astrophysics Research, University of Hertfordshire, College Lane, Hatfield AL10 \\ $9 \mathrm{AB}, \mathrm{UK}$ \\ ${ }^{4}$ Departament de Física Aplicada, Universitat Politècnica de Catalunya, c/Esteve Terrades 5, \\ 08860 Castelldefels, Spain \\ ${ }^{5}$ Astrophysics Research Institute, Liverpool John Moores University, 12 Quays House, Birken- \\ head, CH41 1LD, UK
}

DOI: will be assigned

\begin{abstract}
The evolution of white dwarfs is a simple gravothermal process. This process can be tested in two ways, through the luminosity function of these stars and through the secular variation of the period of pulsation of those stars that are variable. Here we show how the mass of the axion can be constrained using the white dwarf luminosity function.
\end{abstract}

\section{Introduction}

White dwarfs are the final remnants of low- and intermediate-mass stars [1. Since their structure is dominated by the pressure of partially or strongly degenerate electrons, they cannot obtain energy from the thermonuclear burning processes and their evolution can be described, in a first approximation, as a simple gravothermal process. The white dwarf cooling rate can be measured in two ways, using their luminosity function or employing the secular variation of the period of pulsation in the case of variable white dwarfs.

An important property of the luminosity function of white dwarfs is that the shape of the bright branch is independent of the star formation rate and provides a direct measurement of the mass averaged characteristic cooling time [2. This property has provided some hint that axions of the DFSZ type could exist and should have a mass in the range of $\sim 4-8 \mathrm{meV}$, but not much larger [3, 4, [5].

\section{The luminosity function}

The white dwarf luminosity function has been noticeably improved with the data provided by large sky surveys. Figure 1 right, displays the luminosity function obtained from the SDSS with white dwarfs identified from their proper motion [6] (HA-LF) and the one constructed with the same method but limited to spectroscopically-identified DA white dwarfs 77 (DG-LF). The monotonic behavior of this function clearly proves that the evolution of white dwarfs is a 

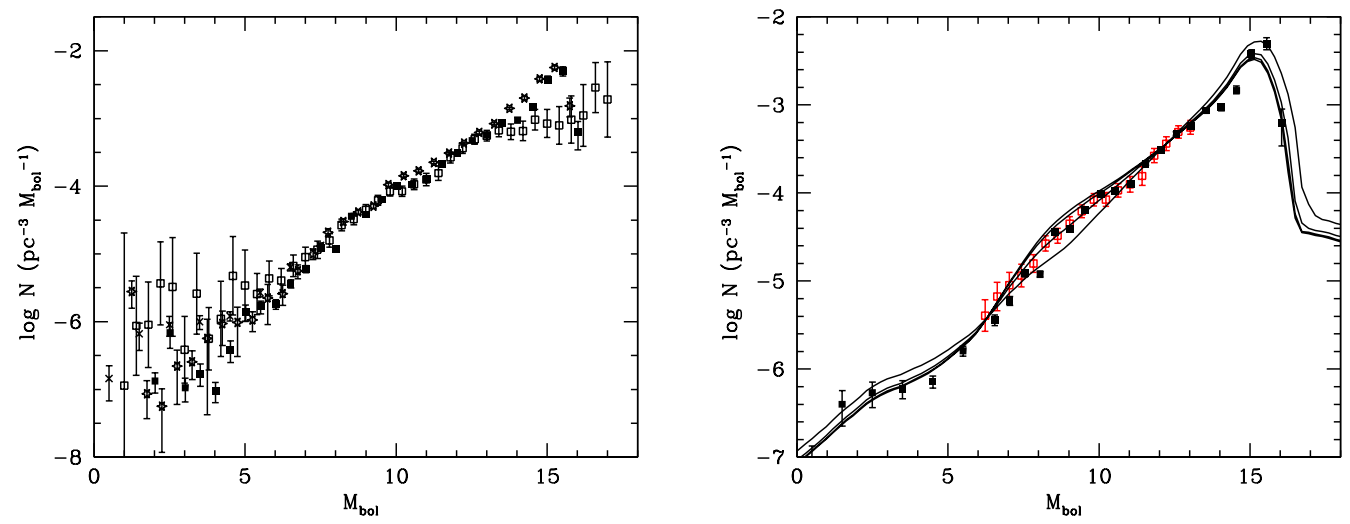

Figure 1: Left: Luminosity functions obtained from large surveys. Solid squares: SDSS, all spectral types [6], hollow squares: SDSS, only DA white dwarfs [7, crosses: SDSS, hot DA white dwarfs [8], stars: SuperCOSMOS Sky Survey [9]. Right: Luminosity functions obtained assuming an axion electron coupling (up to down) $g_{\mathrm{ae}} \times 10^{13}=0.0,1.12,2.24,4.48$. Black squares: extended luminosity function, red hollow squares: pure DA luminosity function, see text for details.

simple gravothermal process, while the sharp cut-off shown by the HA-LF distribution at low luminosities is the consequence of the finite age of the Galaxy. The discrepancies between both at low luminosities are well understood and can be attributed to the different way in which the effective temperatures and gravities of the sample have been determined 7 . Furthermore, the DG-LF only considers DA white dwarfs and, at low temperatures, it is hard to separate these stars from non-DA white dwarfs. For this reason the DG-LF has to be limited to magnitudes smaller than $M_{\mathrm{bol}} \sim 13$. At high luminosities, $M_{\mathrm{bol}}<6$, the dispersion of both functions is considerably large. The reason is that both luminosity functions have been built using the reduced proper motion method which is not appropriate for bright white dwarfs. The UV-excess method has allowed to build a luminosity function for magnitudes ranging from -0.75 to 7 (KZLF) 8. This method, however, is not adequate for dim stars and becomes rapidly incomplete out of this range of magnitudes. Since this sample overlaps with the HA-LF, it is possible to extend the luminosity function to the brightest region assuming continuity and just retaining

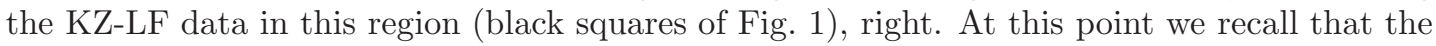
process of formation of white dwarfs is not well known and that their structure is somewhat dependent on the initial conditions until neutrino losses have finished to erase the memory of these conditions. This happens when $M_{\text {bol }} \sim 6$ or, equivalently, when $\log \left(L / L_{\odot}\right) \sim-0.5$. Therefore, the use of very hot white dwarfs for diagnostic purposes has to be taken with some care.

Since the integration time of the SDSS catalogue is fixed, the S/N ratio depends on the brightness of the source and this can lead to large uncertainties in the determination of the parameters of faint white dwarfs with the consequent risk of introducing systematic errors. Fortunately, a completely independent luminosity function has been recently obtained [9] from 
the SuperCOSMOS Sky Survey (hereafter called RH-LF) that does not excessively deviate from the HA-LF at low luminosities. This means that these luminosity functions are probably not affected by large systematic effects. Additionally, it also indicates that neither the shape of the peak nor the cut-off are yet well constrained. It is important to notice here that in this region the luminosity function strongly depends on the star formation rate. Furthermore, since the RH-LF uses the proper motion method to cull white dwarfs, the bright branch of this luminosity function suffers from the same drawbacks as the HA-LF and the DG-LF luminosity functions.

\section{Results and conclusions}

Figure 1 tight, displays the theoretical luminosity function computed with the method described in Ref. [2, but using updated evolutionary sequences for DA white dwarfs [10. As can be seen, the computed luminosity function reproduces reasonably well the region where the slope only depends on the characteristic cooling time except in the region $M_{\mathrm{bol}} \sim 10$, where the computed value predicts an excess of white dwarfs. This region corresponds to the region where neutrino emission has already stopped and axion emission, if included, still persists. If we restrict ourselves to the region where the luminosity function of DAs is reliable (red squares in Fig. 11), right, the best fit occurs for $g_{\mathrm{ae}} \sim 2.2 \times 10^{-13}$ and is clearly not satisfactory for $g_{\text {ae }}>5 \times 10^{-13}$. This effect is tiny and could be due to many conventional effects (like the details of envelope modelling, or the choice of the initial to final mass relationship, IMF, or metallicity, or even to a recent burst of star formation [4]). All these alternatives need to be systematically examined.

Recently, it has been claimed [11] that the inclusion of the axion emission distorts the thermal profile of white dwarfs and this weakens the ability of the luminosity to bound the mass of axions. The change of the thermal profile is a well-known effect produced by any important emission process, and this is indeed what occurs in the case of bright white dwarfs and/or large axion masses. However, these white dwarfs are not appropriate for diagnostic purposes and here we only consider axions with rather small masses. Thus, the approach adopted here is enough for our purposes. In any case, the corresponding bounds do not differ much, and the differences can possibly be attributed to the different way in which the luminosity function is computed.

In this sense, the new measurement of the secular evolution of the pulsation period of G117B15A 12] has provided an additional support to the hypothesis that white dwarfs are cooling at a rate larger than expected, and that axions could be the responsible of this [13, 14. However, a recent detailed analysis [15] suggests that the coupling constant necessary to account for the observations is $g_{\mathrm{ae}}=4.9 \times 10^{-13}$ or equivalently $m_{\mathrm{a}} \approx 17 \mathrm{meV}$. Recently, it has been found [16] that the rate of period change of the variable white dwarf R548 (ZZ Ceti itself) yields $g_{\mathrm{ae}}=4.5 \times 10^{-13}$ or $m_{\mathrm{a}} \approx 16 \mathrm{meV}$, a very similar result. These values are larger than the upper bound obtained from the luminosity function and could also be in conflict with other bounds like SN1987A [17. Nevertheless, in all cases, the uncertainties are still large enough to prevent a definite conclusion and an improvement of both the models and observational data is necessary.

Patras 2012 


\section{Acknowledgments}

This work has been supported by the MICINN grants AYA2011-24704-1839/ESP and AYA201123102, by the ESF EUROCORES Program EuroGENESIS (MICINN grant EUI2009-04170 and 04167), by SGR grants of the Generalitat de Catalunya and by the European Union FEDER funds.

\section{References}

[1] L.G. Althaus, A.H. Córsico, J. Isern, \& E. García-Berro, "Evolutionary and pulsational properties of white dwarfs", Astron. \& Astrophys. Rev., 18, 47 (2010)

[2] J. Isern, \& E. García-Berro, "White dwarfs as physics laboratories: the axion case", MSAI, 79, 545 (2008)

[3] J. Isern, E. García-Berro, S. Torres, \& S. Catalán, "Axions and the cooling of white dwarf stars", ApJ, 682, L109 (2008)

[4] J. Isern, S. Catalán, E. García-Berro, \& S. Torres, "Axions and the white dwarf luminosity function", J. Phys.: Conf. Ser., 172, 012005 (2009)

[5] J. Isern, L. Althaus, S. Catalán, A. Córsico, E. García-Berro, M. Salaris \& S. Torres, "White dwarfs as physics laboratories: the case of axions", 7th Patras Workshop on Axions, WIMPs and WISPs (PATRAS2011),DESY-PROC-2011, Ed. K. Zioutas \& M. Shumann, p. 158, ArXiv: 1204.3565 (2012)

[6] H.C. Harris et al., "The white dwarf luminosity function from Sloan Digital Sky Survey Imaging data", AJ, 131, 571 (2006)

[7] S. De Gennaro, et al., "White dwarf luminosity and mass functions from Sloan Digital Sky Survey Spectra" AJ, 135, 1 (2008)

[8] J. Krzesinski, et al., "A hot white dwarf luminosity function from the Sloan Digital Sky Survey", A\&A, 508, 339 (2009)

[9] N. Rowell, N.C. Hambly, "White dwarfs in the SuperCosmos Sky Survey: the thin disc, thick disc and spheroidal luminosity functions", MNRAS, 417, 93 (2011)

[10] M. Salaris et al., "A large stellar evolution database for population synthesis studies. VI. White dwarf cooling sequences", ApJ, 716, 1241 (2010)

[11] B. Melendez, M. Miller Bertolami, L. Althaus, "Revisiting the impact of axions in the cooling of white dwarfs", arXiv:1210.0263 (2012)

[12] S.O. Kepler, "White dwarf stars: Pulsations and Magnetism", in Progress in solar/stellar physics with helio- and asteroseismology, ASP Conf. Ser., 462, 322 (2012)

[13] J. Isern, M. Hernanz, \& E. García-Berro, "Axion cooling of white dwarfs", ApJ, 392, L23 (1992)

[14] J. Isern, E. García-Berro, L.G. Althaus, \& A.H. Córsico, "Axions and the pulsation periods of variable white dwarfs revisited", A\&A, 512, A86 (2010)

[15] A.H. Córsico et al., "The rate of cooling of the pulsating white dwarf star G117-B15A: a new asteroseismological inference of the axion mass", MNRAS 424, 2792 (2012)

[16] A.H. Córsico et al., "An independent limit on the axion mass from the variable white dwarf star R548", JCAP, in press, ArXiv 1211.3389 (2012)

[17] G.G. Raffelt, J. Redondo, N.V. Viaux, "The meV mass frontier of axion physics", PRD 84, 103008 (2011) 